\title{
Bronchitis and Pneumonia
}

\author{
Manisha Juthani-Mehta, MD and Vincent Quagliarello, MD
}

\section{Key Points}

- The incidence, morbidity, and mortality of both bronchitis and pneumonia are high in older adults and increase with age.

- The clinical manifestations of bronchitis and pneumonia in older adults may not be typical (e.g., cough, fever, dyspnea). Many older adults, particularly nursing home residents, may present with altered mental status and with or without fever. Obtaining a chest X-ray is often critical to make the distinction between the two clinical entities.

- Unique risk factors for pneumonia in older adults include multiple comorbidities (e.g., COPD, DM, CHF), poor oral hygiene, lack of vaccinations (e.g., S. pneumoniae, influenza), and swallowing difficulty.

- Empirical treatment decisions for pneumonia should be based on site of care. Recent information suggests that in-home therapy for community dwellers and therapy within the nursing home for long-term care residents is feasible with good outcomes in selected patients.

- Prevention strategies for pneumonia should be targeted towards providing vaccinations, improving oral hygiene, and improving swallowing difficulty.

\section{Bronchitis}

\section{Epidemiology and Clinical Relevance}

Chronic bronchitis, which affects most patients with chronic obstructive pulmonary disease (COPD), is characterized by a chronic cough productive of sputum lasting

M. Juthani-Mehta and V. Quagliarello ( $\square)$

Department of Medicine, Section of Infectious Diseases, Yale University School of Medicine, 300 Cedar Street, TAC S-169, New Haven, CT 06520, USA

e-mail: vincent.quagliarello@yale.edu 
more than 3 months of the year for 2 consecutive years. Chronic bronchitis usually results from smoking-related obstructive disease. Although many elderly patients with chronic bronchitis may be ex-smokers, age-related decline in lung function can also precipitate COPD symptoms. In elderly patients with chronic bronchitis, acute exacerbations of chronic bronchitis (AECB) are common. AECB is defined as worsening shortness of breath and cough and/or sputum production that cannot be accounted for by daily variability and requires a change in therapy. AECB can lead to poor quality of life, loss of functional status, hospitalizations, loss of lung function, and more often fatality in elderly patients. Risk factors for AECB include respiratory infection, environmental exposures (e.g., air pollution), and poor compliance with prescribed COPD therapies. Lower respiratory tract infection often triggers AECB accounting for $80 \%$ of all episodes. Viral infections, including influenza, parainfluenza, and rhinoviruses, account for $30 \%$ of exacerbations and are associated with superimposed bacterial infections as well. Streptococcus pneumoniae, Haemophilus influenzae, and Moraxella catarrhalis are the most common pathogens in mild cases of AECB and account for 40-60\% of cases. In more severe cases of AECB requiring mechanical ventilation, enteric gram-negative bacilli including Pseudomonas and Stenotrophomonas spp. have been identified. Atypical pathogens, mostly Chlamydia pneumoniae, have only been implicated in $<10 \%$ of cases (1).

\section{Clinical Manifestations}

In elderly patients, symptoms and signs of AECB include increased sputum production and/or sputum purulence, worsening dyspnea and cough, onset of or worsened fluid retention, fever, tachycardia, and altered mental status. Increasing age and comorbid conditions such as cardiac disease, malnutrition, and underlying severe COPD increases the risk of hospitalization and treatment failure (1).

\section{Diagnostic Tests}

The diagnosis of AECB is often made on clinical findings. However, if there is sufficient sputum production, a sputum specimen for Gram stain and culture can help guide antimicrobial treatment decisions. In elderly patients, where symptoms of AECB may be present but sputum often cannot be produced, empirical treatment should be initiated. A chest radiograph should be obtained, especially if there are abnormal breath sounds to differentiate AECB from pneumonia.

\section{Treatment}

The main goal of treating AECB is to allow for rapid symptom relief, eradication of causative pathogens, decreased likelihood of future recurrence, and preservation 


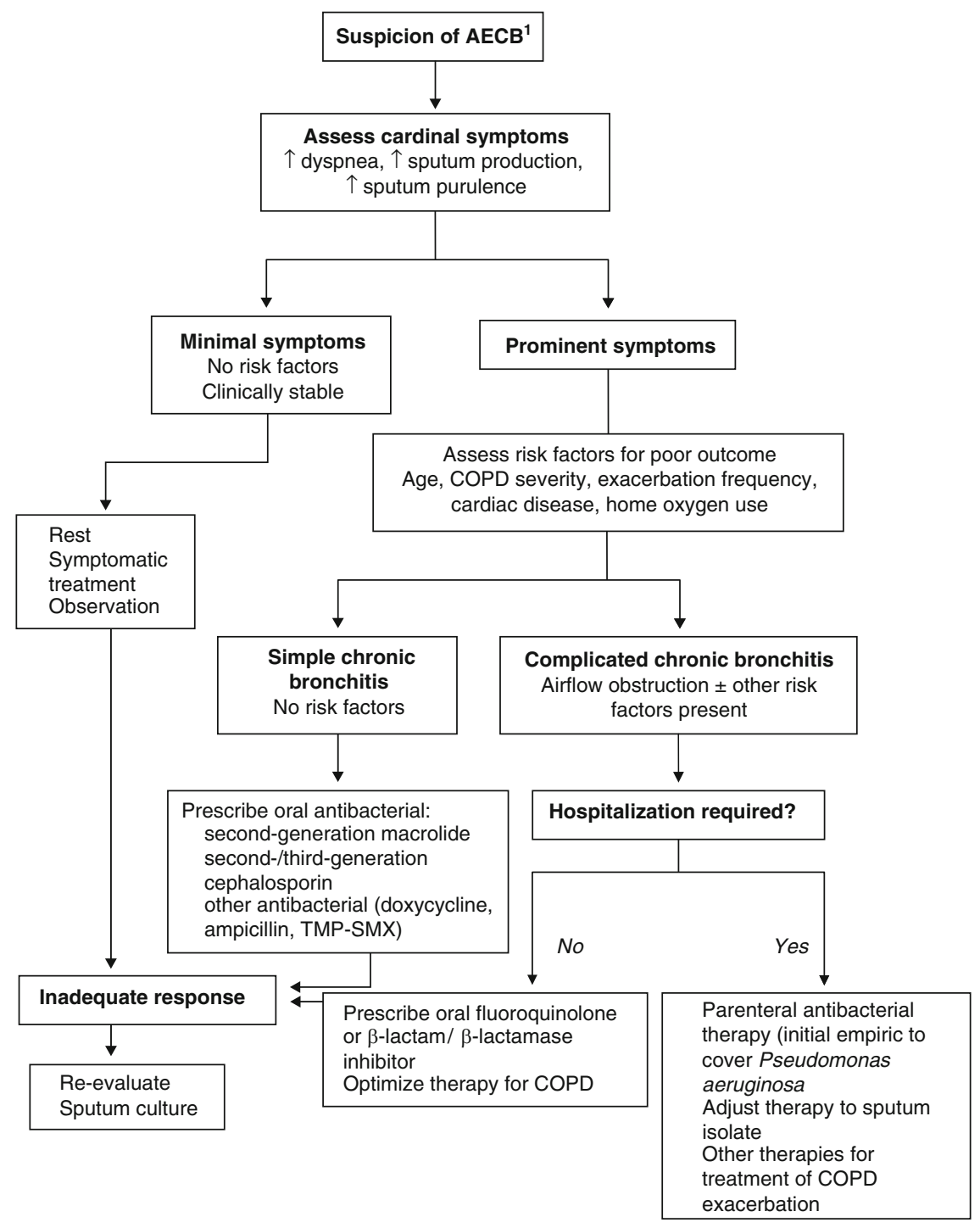

Fig. 1 Algorithm for treatment of acute exacerbations of chronic bronchitis ${ }^{1}$ COPD $=$ chronic obstructive pulmonary disease TMP-SMX = trimethoprim-sulfamethoxazole

of lung function. Early studies of effectiveness of antibacterials as treatment for AECB were inconclusive; however, more recent clinical trials have shown that antimicrobial therapy is effective when administered to risk-stratified groups. An algorithm for empirical treatment decisions in elderly patients with AECB is provided in Fig. 1. Because renal clearance may decline for certain antibiotics with age, some elderly patients may need to be treated as if they have chronic renal insufficiency. Other therapeutic interventions include supplemental oxygen if needed, bronchodilators, rest, fluids, optimized nutrition, judicious use of corticosteroids, early 
administration of a neuraminidase inhibitor if influenza infection is confirmed, and treatment of right heart failure if present (1).

\section{Prevention}

Pulmonary rehabilitation programs can improve the baseline lung function of elderly patients with chronic bronchitis thereby preventing future episodes. Smoking cessation is critical and can also lead to the attenuation of disease progression. Pneumococcal and influenza vaccines are often administered to prevent both upper and lower respiratory tract infection (1). Further details on vaccinations are presented in the section on Pneumonia Prevention.

\section{Pneumonia}

\section{Epidemiology and Clinical Relevance}

\section{Epidemiology and Classification}

Pneumonia is a leading cause of morbidity and mortality in older adults; the incidence of pneumonia increases with age (2). Almost half of all infectious diseases hospitalizations and deaths are associated with pneumonia in older adults (3). While rates for those aged $\geq 85$ years have remained consistently high, rates of hospitalization for pneumonia among older adults aged 65-84 have increased over time (4). The overall rate of community-acquired pneumonia (CAP) in persons aged 65-69 years ranges from 18.2 cases per 1,000 person-years to 52.3 cases per 1,000 person-years among those aged $\geq 85$ years, resulting in an estimate of 915,900 episodes per year of CAP in adults $\geq 65$ years in the United States (2). For residents of nursing homes age 65 years and older, the rate of nursing home acquired pneumonia (NHAP) is as high as 365 cases per 1,000 person-years (5). Pneumonia is classified into four categories: community-acquired pneumonia (CAP), hospitalacquired pneumonia (HAP), nursing home acquired pneumonia (NHAP) or healthcare-associated pneumonia (HCAP), and ventilator-associated pneumonia (VAP). CAP and HAP are differentiated as pneumonia that develops while a patient is in an outpatient or inpatient setting, respectively. VAP is defined as pneumonia arising after the patient has received at least $24 \mathrm{~h}$ of mechanical ventilation. NHAP occurs in nursing home residents that acquire pneumonia while residing in the nursing home. Since an increasing number of patients, especially elderly patients, utilize rehabilitation facilities, outpatient surgical centers, and dialysis centers, pneumonia acquired by patients utilizing these facilities is considered HCAP. The epidemiology of HCAP more closely resembles HAP since these patients are not truly residing in the "community" (6). 


\section{Risk Factors}

For CAP among seniors, age, male sex, COPD, immunosuppression, smoking, cancer, and previous hospitalizations for pneumonia were identified as risk factors (2). Functional status at the time of hospital admission has been shown to be a powerful predictor of mortality (7). Among nursing home residents, inadequate oral care, swallowing difficulty, and lack of influenza vaccine have been shown to be modifiable risk factors for $\operatorname{NHAP}(5,8)$. Malnutrition has also been implicated as a potential risk factor for pneumonia in nursing home residents (9). Specifically, nursing home residents with low serum zinc concentrations had an increased incidence and duration of pneumonia, an increased number of new antibiotic prescriptions, and more days of antibiotic use than residents with normal serum zinc concentrations (10).

\section{Microbiology}

The common etiologies for pneumonia in older adults are dependent on the site of acquisition. The predominant pathogen in seniors in all sites is Streptococcus pneumoniae (11). For CAP that often can be treated in the outpatient setting, the common pathogens include Streptococcus pneumoniae, Mycoplasma pneumoniae, Haemophilus influenzae, Chlamydia pneumoniae and the respiratory viruses (e. g., Influenza A and B, adenovirus, respiratory syncytial virus (RSV), parainfluenza, and human metapneumovirus). Mycoplasma pneumoniae and Chlamydia pneumoniae are common causes of CAP in younger adults, but it is unknown how prevalent these pathogens are in the elderly. When the severity of CAP is greater and requires inpatient therapy, the above listed pathogens, Legionella species and Staphylococcus aureus secondary infection during influenza pneumonia, should be considered (12). For patients with HCAP, Staphylococcus aureus (both methicillin-susceptible and methicillin-resistant) was identified in a large proportion of patients (6). Gramnegative bacteria pneumonia (e.g., Pseudomonas species, Klebsiella species) is more common in HAP, NHAP, VAP, and in community-dwelling older adults with preexisting lung disease, particularly those with high aspiration risk. In outbreak settings, Legionella pneumophila, Chlamydia pneumoniae, RSV, influenza, and parainfluenza should be strongly considered (11).

Other pathogens to consider in older adults include mycobacterial diseases. Reactivation of Mycobacterium tuberculosis is three to four times higher in nursing home residents than in community dwellers. Mycobacterium avium intracellulare complex is the causative agent in many evolving, destructive pulmonary infections often in nonsmoking older women who present with cough, fatigue, fever, weight loss, and nonspecific pulmonary infiltrates. Human immunodeficiency virus (HIV) infection with opportunistic pulmonary infections such as Pneumocystis jiroveci (formerly known as carinii) pneumonia, tuberculosis, cryptococcosis, have been increasingly reported in older adults. With advances in highly active antiretroviral therapy, HIV-infected individuals are living longer, but now older adults often 
develop opportunistic pulmonary infections that are associated with HIV treatment failure (see also Chapter "Human Immunodeficiency Virus/Acquired Immunodeficiency Syndrome"). Other opportunistic infections such as Nocardia asteroides and pulmonary aspergillosis should be considered in older adults with malnutrition, progressive weight loss, and evolving pulmonary infiltrates (11).

\section{Clinical Manifestations}

\section{Pathophysiology}

The primary mechanism of pneumonia, for both CAP and NHAP, is bronchoaspiration. Approximately half of all healthy adults aspirate small amounts of oropharyngeal secretions during sleep. Since most healthy adults are colonized with few virulent pathogens in their oropharynx and since forceful coughing, intact ciliary transport, and normal humoral and cellular immune mechanisms are present, healthy adults are often protected from repeated episodes of pneumonia. However, in older adults, there is a higher frequency of silent aspiration, particularly in those with dementia and stroke. Physiologic changes such as a decrease in elastic recoil of the lung, a decrease in compliance of the chest wall, a decrease in respiratory muscle strength, calcification within the rib cage, osteoporosis and resultant vertebral fractures, decreased mucociliary clearance rates, and swallowing difficulty are all associated with aging. In addition, older adults tend to be colonized in the upper respiratory tract with more virulent pathogens such as Enterobacteriaceae, Pseudomonas aeruginosa, and Staphylococcus aureus (11). The combination of higher rates of aspiration, impaired airway defense mechanisms, and colonization with more pathogenic organisms make older adults at greater risk for the development of pneumonia.

During pneumonia, acute inflammation is characterized by an accumulation of neutrophils and plasma exudate in alveolar spaces. In pulmonary capillaries of uninfected lungs, neutrophils remain trapped and ready to respond when needed. During pulmonary infection, neutrophils migrate into alveolar spaces and kill microbes primarily through phagocytosis. Many plasma proteins present in exudates serve opsonic, bacteriostatic, and microbicidal functions as well during infection. As critical as inflammation is for innate immunity and host defense, it can also directly injure the lungs resulting in acute lung injury (13). In older adults, the inflammatory response itself elicited by highly pathogenic organisms can add to the severity of disease experienced in this population.

\section{Clinical Features}

Cough, sputum production, chills, and pleuritic chest pain are more commonly seen among patients with CAP; elderly nursing home residents with NHAP often present 
with altered mental status and with or without fever. The classic triad of cough, fever, and dyspnea is seen in about $50 \%$ of older patients with CAP. Tachypnea (respiratory rate $>20$ breaths/min) and tachycardia (heart rate $>100$ beats $/ \mathrm{min}$ ) were seen in two-thirds of elderly patients with pneumonia, and these signs may precede other clinical features by 3-4 days. Many elderly patients, particularly those that are chronically debilitated, may present with very subtle clinical manifestations such as an aggravation of preexisting comorbidities (e.g., diabetes, $\mathrm{CHF}$ ), unexplained falls, failure to thrive, and poor appetite (11). In older patients, L. pneumophila is a frequent cause of an atypical pneumonia syndrome presenting with constitutional symptoms myalgias, diarrhea, altered mental status, bradycardia, and hyponatremia. None of these signs or symptoms is specific for L. pneumophila, but they can provide insight into the causative pathogen (11).

\section{Diagnostic Tests}

The diagnosis of CAP is made by having suggestive clinical features (e.g., cough, fever, sputum production, and pleuritic chest pain) with a demonstrable infiltrate on chest radiograph. Physical examination findings (e.g., bronchial breath sounds, rales) are important but less sensitive and specific than chest radiograph findings. Older adults may lack clinical features and/or physical examination findings. Therefore, a chest radiograph is important to differentiate CAP from acute bronchitis in an older adult. Routine diagnostic tests to identify an etiologic agent are optional for outpatients with CAP since most patients do well with empirical antibiotic therapy. Pretreatment blood cultures and an expectorated sputum specimen for Gram stain and culture are standard samples to obtain. However, pretreatment blood cultures only identified a probable pathogen in 5-14\% of patients with CAP. Yield from blood cultures is highest in patients with severe CAP, in which more pathogenic organisms, such as $S$. aureus, $P$. aeruginosa, and other gram-negative bacilli are identified (12). Since these pathogens are more commonly identified in older adults, blood cultures should be part of the standard diagnostic evaluation of an older adult with CAP. The clinical indications for more extensive etiologic diagnostic testing are listed in Table 1. Many diagnostic tests to determine the etiology of pneumonia require sputum production, and often older adults have difficulty producing sputum. Nevertheless, diagnostic testing is particularly important when results may alter antibiotic management decisions, when outbreaks must be identified (e.g., severe acute respiratory syndrome [SARS], influenza, Legionnaire's disease, agents of bioterrorism), and when there are epidemiologic implications (e.g., emergence of resistant pathogens). The clinical utility of sputum culture results is largely dependent on the quality of the sputum specimen and whether the specimen was obtained prior to antimicrobial therapy. When sputum is difficult to obtain, urinary antigen tests for S. pneumoniae and L. pneumophila serogroup 1 can assist in making a diagnosis. These tests have a higher diagnostic yield in patients with the most severe disease. Other advantages of these tests include rapidity of results, ability to detect 
Table 1 Community-acquired pneumonia: clinical indications for more extensive diagnostic testing ${ }^{12}$

\begin{tabular}{|c|c|c|c|c|c|}
\hline Indication & $\begin{array}{l}\text { Blood } \\
\text { culture }\end{array}$ & $\begin{array}{l}\text { Sputum } \\
\text { culture }\end{array}$ & $\begin{array}{l}\text { Legionella } \\
\text { UAT }\end{array}$ & $\begin{array}{l}\text { Pneumococcal } \\
\text { UAT }\end{array}$ & Other \\
\hline Intensive care unit admission & $\mathrm{X}$ & $\mathrm{X}$ & $\mathrm{X}$ & $\mathrm{X}$ & $\mathrm{X}^{\mathrm{a}}$ \\
\hline $\begin{array}{l}\text { Failure of outpatient antibiotic } \\
\text { therapy }\end{array}$ & & $\mathrm{X}$ & $\mathrm{X}$ & $\mathrm{X}$ & \\
\hline Cavitary infiltrates & $\mathrm{X}$ & $\mathrm{X}$ & & & $\mathrm{X}^{\mathrm{b}}$ \\
\hline Leukopenia & $\mathrm{X}$ & & & $\mathrm{X}$ & \\
\hline Active alcohol abuse & $\mathrm{X}$ & $\mathrm{X}$ & $\mathrm{X}$ & $\mathrm{X}$ & \\
\hline Chronic severe liver disease & $\mathrm{X}$ & & & $\mathrm{X}$ & \\
\hline $\begin{array}{l}\text { Severe obstructive/structural lung } \\
\text { disease }\end{array}$ & & $\mathrm{X}$ & & & \\
\hline Asplenia & $\mathrm{X}$ & & & $\mathrm{X}$ & \\
\hline Recent travel (past 2 weeks) & & & $\mathrm{X}$ & & $\mathrm{X}^{\mathrm{c}}$ \\
\hline Positive Legionella UAT result & & $X^{d}$ & NA & & \\
\hline Positive Pneumococcal UAT result & $\mathrm{X}$ & $\mathrm{X}$ & & NA & \\
\hline Pleural effusion & $\mathrm{X}$ & $\mathrm{X}$ & $\mathrm{X}$ & $\mathrm{X}$ & $\mathrm{X}^{\mathrm{e}}$ \\
\hline
\end{tabular}

${ }^{12}$ Reference 12

NA not applicable; UAT urinary antigen test

${ }^{a}$ Endotracheal aspirate if intubated, possibly bronchoscopy or nonbronchoscopic bronchoalveolar lavage.

${ }^{\mathrm{b}}$ Fungal and tuberculosis cultures

${ }^{\mathrm{c}}$ Specific tests for exposure to unique pathogens (e.g., avian influenza, SARS, Hantavirus, Burkholderia pseudomallei)

${ }^{\mathrm{d}}$ Special media for Legionella

${ }^{\mathrm{e}}$ Thoracentesis and pleural fluid cultures

antigen even after initiation of antibiotic therapy, and high specificity. For Legionella, all commercially available urinary antigen assays only detect serogroup 1 . Although serogroup 1 accounts for $80-95 \%$ of community-acquired cases of Legionnaire's disease, other serogroups would not be detected by the standard urinary antigen assay (12). For influenza A and B, adenovirus, RSV, parainfluenza, and human metapneumovirus, a direct fluorescent antibody test can be performed rapidly on nasopharyngeal samples and can assist in determining whether antiviral or antimicrobial therapy is warranted. For patients with HAP that require mechanical ventilation, postintubation tracheal aspiration has been shown to be reliable for microbiologic diagnosis when compared with bronchoalveolar lavage (BAL), plugged telescoping catheter, and protected specimen brush procedures (14).

\section{Treatment}

\section{Predicting Clinical Outcome}

Particularly in older adults, prognostic scoring systems can help predict outcomes of therapy. Most scoring systems predict mortality; however, few assess other clinical outcomes such as physical functional ability, cognitive ability, need for nursing home 
care, and overall quality of life which are important outcomes for older adults (15). The pneumonia severity index (PSI) derived and validated by Fine et al. is a two-step 20 variable scoring system that is best at identifying patients that can be treated as outpatients. However, many older adults fall into the high-risk groups purely based on their age; therefore, it is not as useful at predicting treatment outcomes in this group of patients (15). Lim et al. derived and validated CURB65, a modified version of the scoring system of the British Thoracic Society. This system consists of five parameters: confusion, uremia, respiratory rate, low blood pressure, and age $\geq 65$ years (16). CURB65 has been shown to be useful in the emergency department because of its simplicity of use and ability to identify low-risk patients (17). Modifications to CURB65 to account for age $\geq 85$ years and higher blood urea levels further identify those of the older cohort with severe pneumonia (18). In one scoring system derived and validated among seniors $\geq 65$ years of age, five features were independently predictive of hospital mortality: age $>85$ years, impaired motor response, creatinine level of $>1.5 \mathrm{mg} / \mathrm{dL}$, presence of coexisting disease, and extremely abnormal vital signs (19). In all of these scoring systems, age $>85$ years has been shown to be the greatest predictor of mortality. Currently, no scoring systems exist that predict outcomes other than mortality in the care of older adults with pneumonia but several observational studies in nursing home populations document a decline in function after pneumonia. In a prospective study of nursing home residents with lower respiratory tract infection, the incidence in decline of functional status was $29 \%$. Those that had a decline in functional status at 30 days after pneumonia episode were less likely to recover to their baseline status at 90 days (20). Similarly, in a large prospective trial of older adults, functional decline occurred more rapidly among subjects who developed infection and there was a dose response with an increased risk of decline as the number of infectious episodes increased (20).

\section{Site of Care}

Prognostic scoring systems, particularly CURB-65, may help easily identify those patients that can be treated as outpatients. However, for patients with CURB-65 scores $\geq 2$, hospitalization or intensive in-home health care services are usually warranted. Patients with septic shock requiring vasopressors or with acute respiratory failure requiring intubation and mechanical ventilation are usually directed to admission in the intensive care unit (12). However, among older adults, assessing goals of care are often warranted before initiating hospital and/or intensive care unit (ICU admission). For patients with CAP, hospital-at-home services have been shown to be feasible, safe, efficacious, and more cost effective than hospital admission (21). In addition, hospital-at-home has been associated with lower levels of family member stress and does not appear to shift the burden of care from hospital staff to family members (22). ICU admission is not warranted in the older adult in whom comfort is the main goal of care. For nursing home residents with NHAP, a randomized controlled trial of a clinical pathway for treatment of NHAP in the nursing home versus hospitalization resulted in comparable clinical outcomes, 
while reducing hospitalizations and health care costs. In the clinical pathway group, 34 of $327(10 \%)$ were hospitalized while 76 of $353(22 \%)$ were hospitalized in the usual care group, resulting in greater than $50 \%$ proportional reduction in hospitalizations. The mortality rate was $8 \%$ (24 deaths) in the clinical pathway group versus 9\% (32 deaths) in the usual care group. There were no significant differences in quality of life or functional status between the two groups. The overall cost savings by utilizing the clinical pathway per resident was $\$ 1,016$ (95\% CI, \$207-\$1,824) (23). Although this study demonstrated that treatment of pneumonia in the nursing home can reduce hospitalizations and cost, only those residents that met each of the following criteria could be treated in the nursing home for study purposes: pulse of $100 / \mathrm{min}$ or less, respiratory rate of less than $30 / \mathrm{min}$, systolic blood pressure of at least $90 \mathrm{~mm} \mathrm{Hg}$, oxygen saturation of at least $92 \%$ (or $\geq 90 \%$ if the resident had COPD), and the ability to eat and drink. If any one of these criteria was not met, the resident was transferred to the hospital (23). Additionally, because this study was conducted in Canada, and since the United States has a different healthcare financing system, generalizing this clinical pathway to treat pneumonia in the nursing home may not be feasible in the United States. Nevertheless, this study demonstrated proof of principle, and this approach warrants consideration in residents with mild to moderate pneumonia.

\section{Empirical Antibiotic Therapy}

For healthy older adults with no comorbidities, empirical treatment of outpatient CAP can be initiated with a macrolide or doxycycline. However, for those seniors with comorbidities such as chronic heart, lung, liver, or renal disease, diabetes mellitus, alcoholism, or immunosuppression, a respiratory quinolone (e.g., moxifloxacin, levofloxacin) or a $\beta$-lactam plus a macrolide should be initiated for outpatient or inpatient CAP treatment. Retrospective data suggest that patients with severe CAP treated with a $\beta$-lactam plus a macrolide may have improved clinical outcomes compared with those patients treated with a respiratory quinolone (24). If Pseudomonas aeruginosa is a concern, an antipneumococcal, antipseudomonal $\beta$-lactam (e.g., piperacillin-tazobactam, cefepime, imipenem, meropenem) should be considered. Double coverage with ciprofloxacin or levofloxacin can be considered as well. Aminoglycosides are often recommended for double coverage of Pseudomonas; however, in older adults with a decreased glomerular filtration rate, aminoglycosides should be administered cautiously because of nephrotoxic effects. If community-acquired MRSA is a consideration, vancomycin or linezolid can be added (12).

For nursing home residents or older adults with HAP, HCAP, or VAP, antipseudomonal coverage and MRSA coverage may be warranted empirically. Therefore, a common empirical regimen for treatment of HAP, HCAP, or VAP is piperacillintazobactam and vancomycin. However, drug-resistant $S$. pneumoniae has become increasingly prevalent. Age $\geq 65$ years has been identified as a risk factor for $\beta$-lactam-resistant $S$. pneumoniae. Resistance to penicillin and cephalosporins 
appears to be decreasing but macrolide resistance continues to increase. Repeated use of fluoroquinolones predicts an increased risk of infection with fluoroquinoloneresistant pneumococci (12). Because nursing home residents in particular have usually been exposed to multiple courses of antibiotic therapy, they have a higher risk for harboring drug-resistant pneumococci, gram negatives, and S. aureus. If empirical therapy with piperacillin-tazobactam and vancomycin results in treatment failure, vigorous attempts should be made to identify a causative pathogen and broadening antibiotic coverage to a carbapenem may be warranted. For those nursing home residents that are not transferred to a hospital facility and are empirically treated for NHAP at the nursing home, intramuscular once-daily cefepime has been shown to have clinical success and be more cost-effective than ceftriaxone. Of 61 participants (32 received cefepime, 29 received ceftriaxone) that could be evaluated, clinical success occurred in $78 \%$ of cefepime- and $66 \%$ of ceftriaxone-treated patients $(P=0.39)$. Mean antibiotic costs were $\$ 117 \pm 40$ for cefepime- and $\$ 215 \pm 68$ for ceftriaxone-treated patients $(P<0.001)(25)$. For those residents in whom empirical ceftriaxone therapy would be used, intramuscular cefepime at the nursing home can be considered. If treatment in the nursing home setting is preferred with an oral antimicrobial agent, then ciprofloxacin can be administered to cover most of the causative pathogens.

\section{Pathogen-Directed Therapy}

Once the etiology of pneumonia is identified, antimicrobial therapy should be directed at that pathogen. A list of preferred and alternative antimicrobial agents for the most common pathogens identified in older adults is provided in Table 2 (12). Since many older adults are colonized with more drug-resistant organisms, extended spectrum $\beta$-lactamase (ESBL)-producing organisms have been increasingly isolated. Ertapenem, a newer carbapenem with narrower spectrum of activity than meropenem or imipenem, has been shown to have clinical utility in VAP in older adults (26).

\section{Prevention}

The mainstay for the prevention of pneumonia in older adults is pneumococcal and influenza vaccination. As per the Centers for Disease Control and Prevention guidelines, all persons age 65 or older should receive pneumococcal vaccine once. Revaccination is not recommended unless the first dose was administered under the age of 65 in which case revaccination should occur at age 65 if 5 years have passed since vaccination. Since $90 \%$ of all influenza-related deaths occur among people aged at least 65 years, yearly influenza vaccination has been recommended; however, the actual benefits of influenza vaccination in older adults are controversial. Even as vaccination coverage increased from 15 to $65 \%$, 


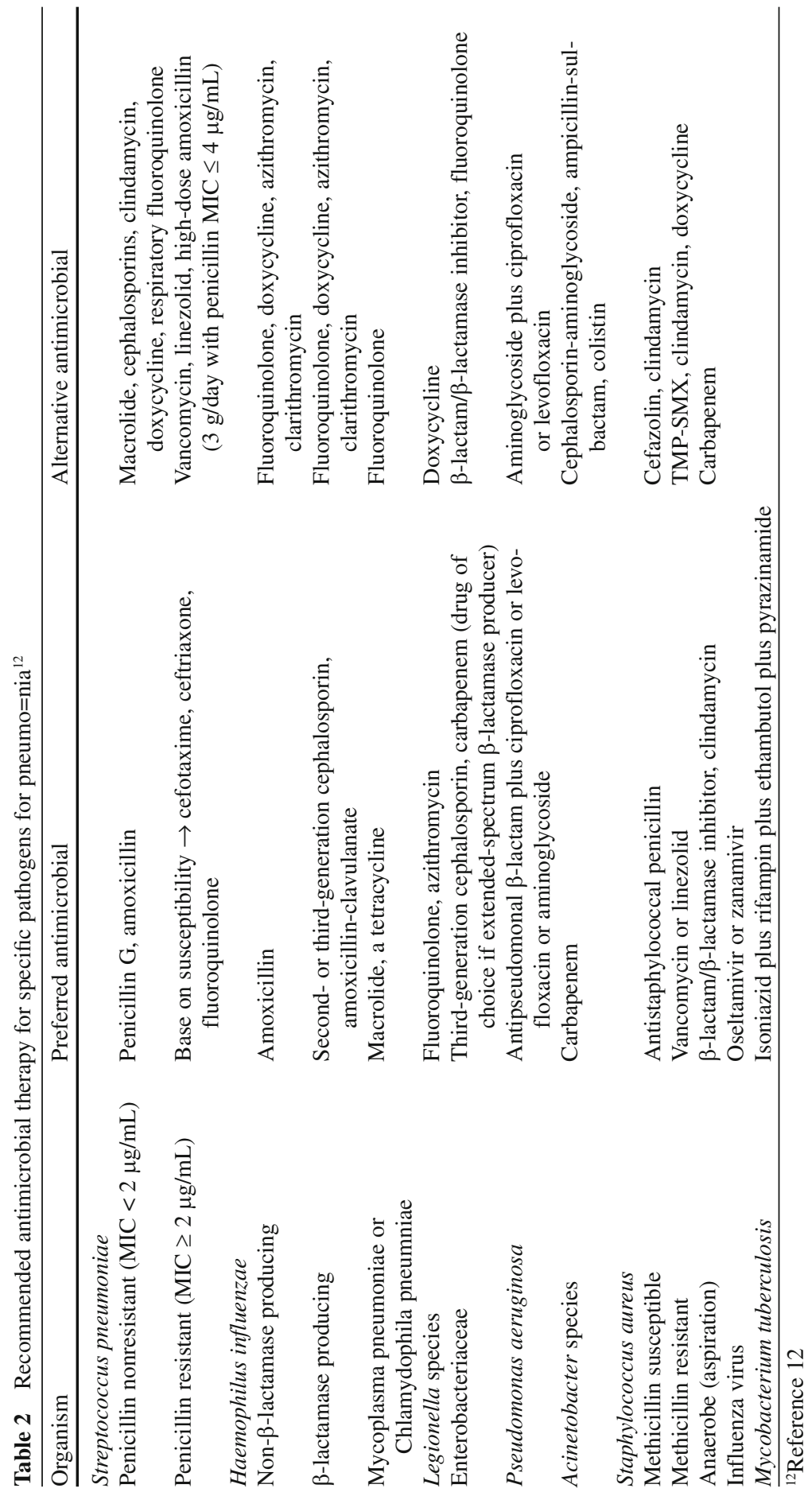


recent excess mortality studies did not demonstrate a decline in influenza-related mortality since 1980 (27). Impaired antibody responses are thought to contribute to stable mortality rates. Booster influenza vaccines have been shown to augment antibody responses among nursing home residents with an impaired response to initial vaccination; however, improved seroprotection rates were not shown to decrease hospitalization rates, death, or antibiotic use in a year without circulating influenza (28). One possible explanation for poor responsiveness to influenza vaccine is age-related impairment in innate immunity, specifically Toll-like receptor (TLR)-mediated expression of the B7 costimulatory molecules CD80 and CD86 which are critical for vaccine immunity (29). Therefore, only yearly influenza vaccination is currently recommended, but future attempts at improved vaccine strategies are warranted.

Since inadequate oral care and swallowing difficulty have been identified as independent modifiable risk factors for NHAP, interventions targeted towards improving oral hygiene and swallowing (e.g., mechanical oral care, $0.12 \%$ oral chlorhexidine rinsing) need to be tested to determine whether they can reduce the incidence of pneumonia. In a recent pilot evaluation in two nursing homes, it was demonstrated that mechanical oral care (i.e., teeth and/or denture and oral cavity brushing) plus $0.12 \%$ oral chlorhexidine rinses were feasible to perform by certified nurses aides, were adhered to by nursing staff, and were effective in improving oral hygiene (i.e., reduction in oral plaque scores) and swallowing difficulty (Vincent Quagliarello, 2009). Additional interventions to improve swallowing need to be investigated as well. The traditional Chinese herbal medicine Banxia Houpu Tang (BHT) found to improve swallowing reflex in patients with stroke and Parkinson's disease has been tested in a randomized controlled trial among elderly nursing home residents and was found to reduce the risk of pneumonia and pneumoniarelated mortality (30). Likely, a combination of interventions to improve oral hygiene and swallowing difficulty will have the greatest potential of reducing the burden of pneumonia in nursing home residents. Future studies will determine whether a similar strategy will reduce the burden of pneumonia in elderly community dwellers.

\section{References}

1. Hayes, D. Jr., \& Meyer, K. C. (2007). Acute exacerbations of chronic bronchitis in elderly patients: pathogenesis, diagnosis and management. Drugs Aging, 24(7), 555-572.

2. Jackson, M. L., Neuzil, K. M., Thompson, W. W., et al. (2004). The burden of communityacquired pneumonia in seniors: results of a population-based study. Clinical Infectious Diseases, 39(11), 1642-1650.

3. Curns, A. T., Holman, R. C., Sejvar, J. J., Owings, M. F., \& Schonberger, L. B. (2002). Infectious disease hospitalizations among older adults in the United States from 1990 through 2002. Archives of Internal Medicine, 165(21), 2514-2520.

4. Fry, A. M., Shay, D. K., Holman, R. C., Curns, A. T., \& Anderson, L. J. (2005). Trends in hospitalizations for pneumonia among persons aged 65 years or older in the United States, 1988-2002. Journal of the American Medical Association, 294(21), 2712-2719. 
5. Quagliarello, V., Ginter, S., Han, L., Van Ness, P., Allore, H., \& Tinetti, M. (2005). Modifiable risk factors for nursing home-acquired pneumonia. Clinical Infectious Diseases, 40(1), 1-6.

6. Kollef, M. H., Shorr, A., Tabak, Y. P., Gupta, V., Liu, L. Z., \& Johannes, R. S. (2005). Epidemiology and outcomes of health-care-associated pneumonia: results from a large US database of culture-positive pneumonia. Chest, 128(6), 3854-3862.

7. Marrie, T. J., \& Wu, L. (2005). Factors influencing in-hospital mortality in community-acquired pneumonia: a prospective study of patients not initially admitted to the ICU. Chest, 127(4), 1260-1270.

8. Loeb, M., McGeer, A., McArthur, M., Walter, S., \& Simor, A. E. (1999). Risk factors for pneumonia and other lower respiratory tract infections in elderly residents of long-term care facilities. Archives of Internal Medicine, 159(17), 2058-2064.

9. Loeb, M., \& High, K. (2005). The effect of malnutrition on risk and outcome of communityacquired pneumonia. Respiratory Care Clinics of North America, 11(1), 99-108.

10. Meydani, S. N., Barnett, J. B., Dallal, G. E., et al. (2007). Serum zinc and pneumonia in nursing home elderly. American Journal of Clinical Nutrition, 86(4), 1167-1173.

11. Janssens, J. P., \& Krause, K. H. (2004). Pneumonia in the very old. Lancet Infectious Disease, $4(2), 112-124$.

12. Mandell, L. A., Wunderink, R. G., Anzueto, A., et al. (2007). Infectious Diseases Society of America/American Thoracic Society consensus guidelines on the management of communityacquired pneumonia in adults. Clinical Infectious Diseases, 44 (Suppl. 2), S27-S72.

13. Mizgerd, J. P. (2008). Acute lower respiratory tract infection. New England Journal of Medicine, 358(7), 716-727.

14. Clec'h, C., Jaureguy, F., Hamza, L., et al. (2006). Agreement between quantitative cultures of postintubation tracheal aspiration and plugged telescoping catheter, protected specimen brush, or BAL for the diagnosis of nosocomial pneumonia. Chest, 130(4), 956-961.

15. Juthani-Mehta, M., \& Quagliarello, V. J. (2004). Prognostic scoring systems for infectious diseases: their applicability to the care of older adults. Clinical Infectious Diseases, 38(5), 692-696.

16. Lim, W. S., van der Eerden, M. M., Laing, R., et al. (2003). Defining community acquired pneumonia severity on presentation to hospital: an international derivation and validation study. Thorax, 58(5), 377-382.

17. Yan Man, S., Lee, N., Ip, M., et al. (2007). Prospective comparison of three predictive rules for assessing severity of community-acquired pneumonia in Hong Kong. Thorax, 62(4), 348-353.

18. Myint, P. K., Kamath, A. V., Vowler, S. L., \& Harrison, B. D. (2007). Simple modification of CURB-65 better identifies patients including the elderly with severe CAP. Thorax, 62(11), 1015-1016; author reply 1016.

19. Conte, H. A., Chen, Y. T., Mehal, W., Scinto, J. D., \& Quagliarello, V. J. (1999). A prognostic rule for elderly patients admitted with community-acquired pneumonia. American Journal of Medicine, 106(1), 20-28.

20. High, K. P., Bradley, S., Loeb, M., Palmer, R., Quagliarello, V., \& Yoshikawa, T. (2005). A new paradigm for clinical investigation of infectious syndromes in older adults: assessment of functional status as a risk factor and outcome measure. Clinical Infectious Diseases, 40(1), $114-122$.

21. Leff, B., Burton, L., Mader, S. L., et al. (2005). Hospital at home: feasibility and outcomes of a program to provide hospital-level care at home for acutely ill older patients. Annals of Internal Medicine, 143(11), 798-808.

22. Leff, B., Burton, L., Mader, S. L., et al. (2008). Comparison of stress experienced by family members of patients treated in hospital at home with that of those receiving traditional acute hospital care. Journal of American Geriatrics Society, 56(1), 117-123.

23. Loeb, M., Carusone, S. C., Goeree, R., et al. (2006). Effect of a clinical pathway to reduce hospitalizations in nursing home residents with pneumonia: a randomized controlled trial. Journal of the American Medical Association, 295(21), 2503-2510. 
24. Lodise, T. P., Kwa, A., Cosler, L., Gupta, R., \& Smith, R. P. (2007). Comparison of betalactam and macrolide combination therapy versus fluoroquinolone monotherapy in hospitalized Veterans Affairs patients with community-acquired pneumonia. Antimicrobial Agents Chemotherapy, 51(11), 3977-3982.

25. Paladino, J. A., Eubanks, D. A., Adelman, M. H., \& Schentag, J. J. (2007). Once-daily cefepime versus ceftriaxone for nursing home-acquired pneumonia. Journal of American Geriatrics Society, 55(5), 651-657.

26. Bassetti, M., Righi, E., Fasce, R., et al. (2007). Efficacy of ertapenem in the treatment of early ventilator-associated pneumonia caused by extended-spectrum beta-lactamase-producing organisms in an intensive care unit. Journal of Antimicrobial Chemotherapy, 60 (2), 433-435.

27. Simonsen, L., Taylor, R. J., Viboud, C., Miller, M. A., \& Jackson, L. A. (2007). Mortality benefits of influenza vaccination in elderly people: an ongoing controversy. Lancet Infectious Disease, 7(10), 658-666.

28. Gaughran, F., Walwyn, R., Lambkin-Williams, R., et al. (2007). Flu: effect of vaccine in elderly care home residents: a randomized trial. Journal of the American Geriatriacs Society, 55(12), 1912-1920.

29. van Duin, D., Allore, H. G., Mohanty, S., et al. (2007). Prevaccine determination of the expression of costimulatory B7 molecules in activated monocytes predicts influenza vaccine responses in young and older adults. Journal of Infectious Diseases, 195(11), 1590-1597.

30. Iwasaki, K., Kato, S., Monma, Y., et al. (2007). A pilot study of Banxia Houpu Tang, a traditional Chinese medicine, for reducing pneumonia risk in older adults with dementia. Journal of the American Geriatrics Society, 55(12), 2035-2040.

\section{Suggested Reading}

Hayes, D. Jr., \& Meyer, K. C. (2007). Acute exacerbations of chronic bronchitis in elderly patients: pathogenesis, diagnosis and management. Drugs Aging, 24(7), 555-572.

Janssens, J. P., \& Krause, K. H. (2004). Pneumonia in the very old. Lancet Infectious Disease, $4(2), 112-124$.

Mandell, L. A., Wunderink, R. G., Anzueto, A., et al. (2007). Infectious Diseases Society of America/American Thoracic Society consensus guidelines on the management of communityacquired pneumonia in adults. Clinical Infectious Diseases, 44 (Suppl. 2), S27-S72.

Quagliarello, V., Juthani-Mehta, M., Ginter, S., Towle, V., Allore, H., Tinetti, M. (2009). Pilot Testing of Intervention Protocols to Prevent Pneumonia among Nursing Home Residents." Journal of the American Geriatrics Society, in press. 